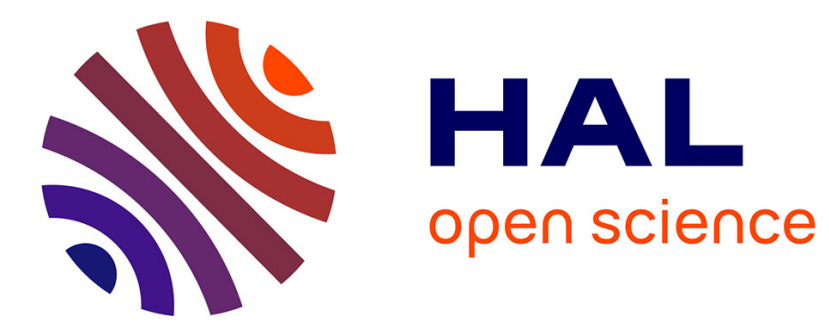

\title{
Sylviculture en forêt tropicale humide guyanaise
} Julien Demenois, Sylvie Gourlet-Fleury, M. Fuhr, Jean-Gaël Jourget

\section{To cite this version:}

Julien Demenois, Sylvie Gourlet-Fleury, M. Fuhr, Jean-Gaël Jourget. Sylviculture en forêt tropicale humide guyanaise. Revue forestière française, 2003, 55 (sp), pp.273-290. 10.4267/2042/5778 . hal03449491

\section{HAL Id: hal-03449491 \\ https://hal.science/hal-03449491}

Submitted on 25 Nov 2021

HAL is a multi-disciplinary open access archive for the deposit and dissemination of scientific research documents, whether they are published or not. The documents may come from teaching and research institutions in France or abroad, or from public or private research centers.
L'archive ouverte pluridisciplinaire HAL, est destinée au dépôt et à la diffusion de documents scientifiques de niveau recherche, publiés ou non, émanant des établissements d'enseignement et de recherche français ou étrangers, des laboratoires publics ou privés. 


\title{
L'HOMME ET LA FOHÊT
}

\section{SYLVICULTURE EN FORÊT TROPICALE HUMIDE GUYANAISE}

\author{
Julien Demenois - Sylvie Gourlet-Fleury - Marc Fuhr - Jean-GaËl Jourget
}

Il est sans doute prétentieux de parler de sylviculture en forêt tropicale humide, du moins dans le sens communément accepté par les forestiers européens. Non par manque de techniques sylvicoles à disposition des forestiers tropicaux, mais par manque à la fois de financements et de certitude sur le devenir à long terme des peuplements traités. Cet article vise toutefois à montrer que, sur ce dernier point, nos connaissances progressent. Il s'appuie pour cela sur la présentation des résultats issus des travaux menés par le CIRAD-Forêt et l'ONF en Guyane, depuis près de 20 ans.

\section{BREF HISTORIQUE DES ESSAIS DE SYLVICULTURE EN FORÊT TROPICALE HUMIDE}

On peut dater les premiers essais de sylviculture en forêt tropicale humide de la fin du XIXe siècle en Asie, puis du début du XXe siècle en Afrique, où les forestiers européens ont cherché à adapter les méthodes mises au point en Birmanie, en Inde, en Indonésie et en Malaisie, ellesmêmes adaptées des pratiques européennes. Deux courants de pensée se sont affrontés sur la question de l'enrichissement des peuplements en essences de valeur, l'un prônant la stimulation de la régénération naturelle pré-existante ou à induire (les Anglo-Saxons au Ghana et au Nigeria), l'autre les plantations selon diverses techniques (les Français en Côte d'Ivoire) (Catinot, 1965 a et b). À partir des années 1950, face aux fréquents échecs enregistrés par les opérations d'enrichissement par plantation, les essais sylvicoles ont largement porté sur l'utilisation de la régénération naturelle.

On distingue deux grands types de méthodes s'appuyant sur la régénération naturelle en forêt (Dupuy, 1998) :

- les méthodes de conversion en futaie régulière (exemples : Malayan Uniform System, Tropical Shelterwood System, Uniformisation par le haut). Ces méthodes consistent à procéder à une récolte unique des bois commercialisables et à réaliser des opérations sylvicoles d'amélioration et de régénération (avant ou après l'exploitation) de manière à régulariser progressivement la structure du peuplement autour d'une même classe d'âge. Cependant, la diversité floristique des forêts denses induit une forte résistance à des sylvicultures trop dirigistes. Les différentiels de croissance entre les espèces rétablissent très rapidement une hétérogénéité de structure. Aussi, ces méthodes ont-elles été un échec, montrant qu'en forêt tropicale humide, d'une part les structures irrégulières sont incontournables, d'autre part les interventions sylvicoles doivent être concentrées sur l'existant ;

- les méthodes de conduite de futaie irrégulière (exemples : amélioration des peuplements naturels, Selective Management System, gestion sélective, Celos Management System). Elles reposent sur un système de coupes polycycliques avec un diamètre d'exploitabilité des espèces 
commerciales suffisamment élevé pour conserver un potentiel sur pied assurant la pérennité commerciale du peuplement. Dans les années 1950, de nombreuses méthodes ont été développées, basées sur un inventaire du peuplement exploitable et du peuplement d'avenir ainsi que sur des interventions sylvicoles (éclaircie systématique ou sélective, par abattage ou par dévitalisation ; délianage ; recépage).

Le bilan des essais menés s'avère mitigé. Certaines techniques ont été appliquées à grande échelle, d'autres n'ont pas dépassé le stade des expérimentations. La plupart se sont heurtées avant tout à la lourdeur des travaux de dégagement de la régénération et au manque de moyens humains et financiers sur le long terme sans parler, dans certains pays, de l'augmentation de la pression sur la ressource ligneuse et sur les terres forestières.

Le début des années 1980 a connu un regain d'intérêt pour l'aménagement des forêts naturelles du fait de la remise en cause, au niveau international, des plantations d'essences à croissance rapide, le plus souvent exotiques. Dès la fin des années 1970, le Centre technique forestier tropical (CTFT, aujourd'hui CIRAD-Forêt) a mis en place, en collaboration avec les organismes nationaux de recherche et de gestion forestière, un réseau de dispositifs sylvicoles expérimentaux en Afrique (Côte d'Ivoire, République Centrafricaine, Congo, Gabon), en Amérique latine (Guyane, Brésil) et en Asie (Indonésie).

Tirant leçon des succès et des échecs passés, les méthodes testées sur ces dispositifs étaient simples et de mise en œuvre peu coûteuse. Il s'agissait de stimuler la croissance des jeunes arbres déjà installés en forêt, sans se préoccuper de la régénération, et de limiter les interventions à l'exploitation de gros bois suivie immédiatement d'une éclaircie, celle-ci étant systématique (choix d'espèces ou de diamètres) et non plus dirigée (dégagement des seules espèces de valeur) comme par le passé. Ces principes ont été appliqués en Guyane au dispositif de Paracou.

Toutefois, l'utilisation de règles systématiques d'éclaircie a été remise en cause car elle présente le triple inconvénient :

- d'éliminer les semenciers de nombreuses espèces jugées sans valeur commerciale (risque potentiel pour le maintien de la biodiversité),

- de juger aujourd'hui indésirables des espèces pouvant présenter, à l'avenir, un intérêt particulier,

- d'être inutilement coûteuses, quand elles sont appliquées dans des zones pauvres en espèces de valeur (Gourlet-Fleury, 1990, 1992).

Plus récemment, avec la signature des grandes conventions internationales, de nouvelles préoccupations ont émergé. L'attention s'est portée sur les divers services fournis par la forêt, autres que la simple production de bois d'œuvre : fourniture de produits non ligneux, préservation de la biodiversité, participation aux grands cycles biogéochimiques. L'effort a donc porté sur la mise au point de techniques d'exploitation respectueuses de l'environnement. On a vu apparaître les méthodes d'exploitation à faible impact (Pinard et al., 1995 ; Bertault et Sist, 1997 ; Van der Hout, 1999 ; Durrieu et al., 1998 ; Sist, 2000 ; Van Leersum et al., 2001). Alors que, dans les essais antérieurs, l'exploitation était souvent dissociée de la sylviculture, elle est apparue dès lors comme un outil sylvicole à part entière, à optimiser. Ces considérations ont conduit l'ONF, en Guyane, à mettre en route des expérimentations de sylviculture "mixte" dans les forêts de Risquetout et d'Organabo (Fuhr et Plancheron, 2001).

Aujourd'hui, si le gestionnaire forestier cherche toujours à accélérer la productivité en bois d'œuvre par le renouvellement des tiges d'espèces commerciales et par une croissance plus soutenue de leur volume, il vise avant tout à imiter la nature et à limiter, autant que faire se peut, l'impact potentiellement négatif de ses interventions. 


\section{TRAITEMENTS SYLVICOLES APPLIQUÉS}

\section{DANS LES DISPOSITIFS ${ }^{(1)}$ EXPÉRIMENTAUX DE GUYANE}

Les dispositifs de Guyane couvrent différentes situations forestières, selon que les peuplements n'ont jamais été exploités (forêts dites non perturbées, c'est le cas du dispositif de Paracou), ou qu'ils ont fait l'objet d'exploitations anciennes, avant implantation du dispositif et test de modalités d'intervention (cas des dispositifs de Risquetout et d'Organabo).

\section{Le dispositif expérimental de Paracou}

L'objectif initial du dispositif de Paracou était d'étudier l'effet d'interventions sylvicoles simples et peu coûteuses à mettre en œuvre, susceptibles d'accélérer la reconstitution du peuplement forestier après exploitation. II s'agissait d'établir un "modèle" de sylviculture qui, tout en assurant une production de bois, permette le renouvellement des essences de valeur.

Deux grands types d’intervention ont été réalisés :

- abattage et débardage d'essences de bois d'œuvre ou d'énergie, selon des méthodes traditionnelles ;

- éclaircie par dévitalisation chimique, d'essences dépourvues d'intérêt commercial ou présentant des anomalies, faite selon la technique dite des entailles "malaises" (cf. photo 1, cicontre) avec application d'un produit arboricide.

Photo 1 Technique de dévitalisation dite des “entailles malaises" (Station de Paracou en Guyane) Photo CIRAD-Forêt

Par combinaison des interventions et de leur intensité, 4 types de traitements sylvicoles, répétés trois fois, ont été réalisés sur 12 parcelles de 6,25 ha chacune. Ces traitements sont les suivants :

- Témoin (To) : parcelles témoins, surface terrière moyenne du peuplement de $30 \mathrm{~m}^{2} / \mathrm{ha}$, pour 620 tiges/ha.

- Traitement 1 ( $\left.\mathrm{T}_{1}\right)$ : exploitation, pour le bois d'œuvre, des arbres de diamètre ${ }^{(2)} \geq 50$ ou $60 \mathrm{~cm}$, appartenant à 58 espèces ou groupes d'espèces ; environ 10 arbres à l'hectare ont été prélevés, soit une surface terrière moyenne de $5 \mathrm{~m}^{2} /$ ha.

- Traitement 2 ( T2) : exploitation pour le bois d'œuvre comme en T1, suivie d'une éclaircie par dévitalisation d'arbres sans intérêt commercial potentiel de diamètre $\geq 40 \mathrm{~cm}$, soit environ 30 arbres/ha ; au total, le traitement 2 a prélevé une surface terrière moyenne de $12 \mathrm{~m}^{2} / \mathrm{ha}$.

- Traitement 3 (T3) : exploitation pour le bois d'œuvre comme en T1, complétée d'une exploitation pour le bois énergie d'arbres de diamètre compris entre 40 et $50 \mathrm{~cm}$ (environ 15 arbres/ha) et suivie d'une éclaircie par dévitalisation (environ 20 arbres/ha) ; le traitement 3 a prélevé une surface terrière moyenne de $15 \mathrm{~m}^{2} / \mathrm{ha}$.

(1) Pour la présentation des dispositifs expérimentaux de Silvolab, cf. article Weigel et al., dans ce numéro.

(2) Diamètre à hauteur de poitrine (1,30 m). 


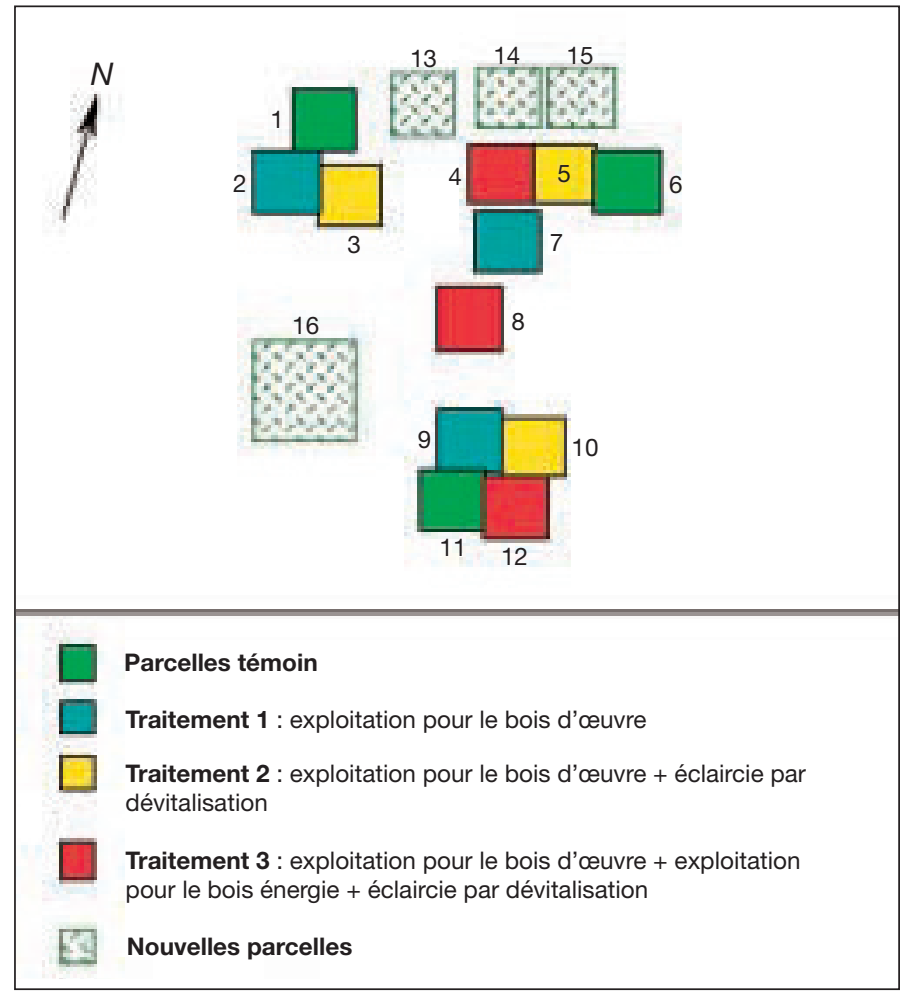

FIGURE 1

\section{DESCRIPTIF DU DISPOSITIF DE PARACOU (CIRAD-Forêt)}

Nota : Chaque parcelle est découpée en 4 carrés délimités par des layons formant un placeau central de 6,25 hectares entouré par une zone tampon ayant pour objectif d'éliminer les artefacts liés aux effets de bordure. Les différents traitements ont été appliqués sur la surface totale des parcelles.

\section{FIGURE 2}




(D désigne le diamètre à hauteur de poitrine, soit $1,30 \mathrm{~m}$ du sol)

\begin{tabular}{|c|c|c|c|}
\hline $\begin{array}{l}\text { Modalité } \\
\text { d’éclaircie }\end{array}$ & $\begin{array}{l}\text { Arbre d'avenir } \\
\text { (AA, ou désigné, } \\
\text { au profit duquel } \\
\text { on travaille) }\end{array}$ & Arbre dévitalisé (AD) & $\begin{array}{l}\text { Surface terrière } \\
\text { éliminée }\end{array}$ \\
\hline MI 1 & $\begin{array}{l}\text { Espèce commerciale }{ }^{(1)} \\
\text { tige de } D_{A A} \geq 12,5 \mathrm{~cm}\end{array}$ & $\begin{array}{l}\text { Espèce non commerciale située dans un rayon } \\
\text { de } 10 \mathrm{~m} \text { de } A A, D_{A D} \geq D_{A A}\end{array}$ & $\begin{array}{l}5,6 \mathrm{~m}^{2} / \mathrm{ha}(24 \%) \\
\text { à Risquetout } \\
4 \mathrm{~m}^{2} / \text { ha }(15 \%) \\
\text { à Organabo }\end{array}$ \\
\hline $\mathrm{Ml} 2$ & $\begin{array}{l}\text { Espèce commerciale, } \\
\text { tige de } D_{A A} \geq 27,5 \mathrm{~cm}\end{array}$ & $\begin{array}{l}\text { Espèce non commerciale située dans un rayon } \\
\text { de } 10 \mathrm{~m} \text { de } A A, D_{A D} \geq D H P_{A A}-5 \mathrm{~cm} \\
+ \text { toute tige d'espèce non commerciale } \\
\text { (sauf Eschweilera spp.) de } \mathrm{DHP}_{A D} \geq 37,5 \mathrm{~cm} \\
\text { (Risquetout) ou } 57,5 \mathrm{~cm} \text { (Organabo), } \\
\text { sur l'ensemble de la parcelle }\end{array}$ & $\begin{array}{l}1,6 \mathrm{~m}^{2} / \mathrm{ha}(7 \%) \\
\text { à Risquetout } \\
3,7 \mathrm{~m}^{2} / \mathrm{ha}(14 \%) \\
\text { à Organabo }\end{array}$ \\
\hline $\mathrm{Ml} 3$ & $\begin{array}{l}\text { Espèce commerciale, } \\
\text { tige de } D_{A A} \geq 27,5 \mathrm{~cm}\end{array}$ & $\begin{array}{l}\text { Espèce non commerciale située dans } \\
\text { un rayon de } 10 \mathrm{~m} \text { de } A A, D_{A D} \geq D_{A A}-20 \mathrm{~cm} \\
\text { avec une limite inférieure: } D H P_{A D} \geq 17,5 \mathrm{~cm} \\
\text { + toute tige d'espèce non commerciale } \\
\text { (sauf Eschweilera spp.) de } D H P_{A D} \geq 37,5 \mathrm{~cm} \\
\text { (Risquetout) ou } 57,5 \mathrm{~cm} \text { (Organabo), } \\
\text { sur l'ensemble de la parcelle }\end{array}$ & $\begin{array}{l}11,6 \mathrm{~m}^{2} / \text { ha }(46 \%) \\
\text { à Risquetout } \\
6,6 \mathrm{~m}^{2} / \text { ha }(27 \%) \\
\text { à Organabo }\end{array}$ \\
\hline
\end{tabular}

(1) Par espèce commerciale, on entend l'une des 51 espèces et groupes d'espèces habituellement commercialisés en Guyane.

\section{Forêts "pilotes" de Risquetout et d'Organabo}

La forêt domaniale de Risquetout (10 ooo ha), proche de 3 chefs-lieux de communes, a fait l'objet de 2 à 3 passages en exploitation depuis le début du XXe siècle, la dernière exploitation ayant été réalisée entre 1980 et 1983. La forêt domaniale d'Organabo (11 360 ha), assez éloignée des centres urbains, n'a été exploitée qu'une seule fois, entre 1986 et 1995 selon les parcelles.

Les dispositifs expérimentaux de Risquetout et d'Organabo ont été mis en place dans le but de répondre à la question suivante : est-il possible, dans des peuplements ayant déjà été exploités, de dynamiser la croissance d'essences commerciales par une éclaircie sylvicole (dévitalisation) pratiquée en cours de révolution ? Ainsi, selon les modalités testées, les éclaircies sylvicoles ontelles été faites entre 8 et 20 ans après la dernière exploitation.

Les règles d'éclaircie testées sont nées d'une réflexion commune entre l'ONF et le CIRAD-Forêt, face à différents inconvénients présentés par les règles systématiques utilisées à Paracou (GourletFleury, 1992). L'idée était de combiner : un travail au profit des arbres d'avenir, afin d'éviter des interventions inutiles dans les zones trop pauvres et une élimination systématique de semenciers de certaines espèces, avec un minimum de règles systématiques d'intervention afin de limiter les décisions subjectives (Gourlet-Fleury, 1990). Les éclaircies ont ainsi été qualifiées de "mixtes" (sélectives/systématiques).

Chaque dispositif comprend 3 groupes de 2 placettes, de 4 ha chacune, soit 24 ha pour chaque forêt. Chaque binôme, implanté à l'intérieur d'une même parcelle, comprend (cf. figure 2, p. 276) :

- une placette traitée selon l'une des modalités décrites ;

- une placette témoin. 
Nous passons en revue, dans les paragraphes qui suivent, les principaux résultats obtenus dans ces trois dispositifs expérimentaux ${ }^{(3)}$ avant de discuter une stratégie possible de sylviculture pour les forêts aménagées de Guyane.

\section{IMPACT DES TRAITEMENTS SYLVICOLES $(4)$}

\section{Croissance dans les peuplements non perturbés}

Toutes essences et tous diamètres confondus, les accroissements moyens en diamètre observés dans les parcelles de Paracou, avant traitement sylvicole, sont compris entre 0,09 et 0,17 cm/an.

FIGURE 3

ACCROISSEMENTS EN DIAMÈTRE PAR CLASSES DE DIAMÈTRE, GROUPES DE PARCELLES ET TOUTES ESSENCES CONFONDUES À PARACOU



Les moyennes sont représentées avec leur intervalle de confiance à $95 \%$.

TABLEAU II

Accroissements moyens en diamètre (cm/an) par classe de diamètre pour les trois essences les plus exploitées en Guyane

[données de Paracou (Gourlet-Fleury, 2000), parcelles témoin To, période 1984-1997]

\begin{tabular}{|c|c|c|c|c|c|c|}
\hline & $10-20 \mathrm{~cm}$ & $20-30 \mathrm{~cm}$ & $30-40 \mathrm{~cm}$ & $40-50 \mathrm{~cm}$ & $50-60 \mathrm{~cm}$ & $\geq 60 \mathrm{~cm}$ \\
\hline $\begin{array}{l}\text { Angélique ............ } \\
\text { (Dycorynia guianensis) }\end{array}$ & 0,15 à 0,20 & 0,24 à 0,31 & 0,22 à 0,34 & 0,21 à 0,32 & 0,14 à 0,37 & 0,06 à 0,27 \\
\hline $\begin{array}{l}\text { Gonfolos ........... } \\
\text { (Qualea rosea } \\
\text { et Ruitzerania albiflora) }\end{array}$ & 0,12 à 0,18 & 0,21 à 0,36 & 0,30 à 0,44 & 0,21 à 0,33 & 0,23 à 0,43 & 0,14 à 0,28 \\
\hline $\begin{array}{l}\text { Grignon franc } . . . . \\
\text { (Sextonia rubra) }\end{array}$ & 0,01 à 0,09 & 0,03 à 0,14 & 0,24 à 0,38 & 0,24 à 0,40 & 0,27 à 0,45 & 0,18 à 0,46 \\
\hline
\end{tabular}

NB: Étant donné la faiblesse des effectifs, pour le Grignon franc, toutes classes de diamètre confondues, et pour l'Angélique et les Gonfolos au-delà de la classe de diamètre 40-50 cm, ces résultats doivent être interprétés avec prudence.

(3) Concernant les forêts "pilotes", seuls les résultats de l'éclaircie mixte 1 sont présentés. Les résultats des éclaircies mixte 2 et mixte 3 sont en cours d'analyse.

(4) En ce qui concerne Paracou, le bilan détaillé des interventions sylvicoles sur la dynamique des peuplements est présenté dans l'ouvrage "Ecology and Management of a Neotropical Rainforest : the lessons of Paracou, French Guiana" / S. Gourlet-Fleury, J.-M. Guehl, O. Laroussinie Eds. - Paris : Elsevier, 2004. 
Dans pratiquement tous les groupes de parcelles (témoin et T1, T2, T3 avant intervention sylvicole), on note une tendance à l'augmentation des accroissements avec la classe de diamètre. Une analyse de variance réalisée groupe par groupe montre un effet très significatif de ce facteur.

Les comparaisons multiples de moyennes isolent systématiquement la classe $10-20 \mathrm{~cm}$ : les accroissements sont significativement plus faibles dans cette classe de diamètre que dans les autres (Gourlet-Fleury, 2000 et figure 3, p. 278).

Les accroissements moyens observés sur les trois essences les plus couramment exploitées en Guyane (Angélique, Gonfolos et Grignon franc) sont présentés dans le tableau II (p. 278).

\section{Effet des interventions sylvicoles sur la croissance}

Si l'on compare, toutes essences confondues, les résultats de Paracou et ceux obtenus dans les forêts "pilotes", il apparaît que l'accroissement est multiplié par un facteur 1,3 à 3 (moyenne sur le peuplement, entre 4 et 12 ans après intervention sylvicole), par rapport aux valeurs enregistrées dans les parcelles témoins (cf. tableau III, ci-dessous).

TABLEAU III

Facteur multiplicatif sur l'accroissement en diamètre en fonction de l'intensité du traitement sylvicole

(Gourlet-Fleury, 2000; Fuhr et al., 2001), nota: To $=1$

\begin{tabular}{|c|c|c|c|c|c|}
\hline & \multicolumn{3}{|c|}{ Paracou } & \multicolumn{2}{|c|}{$\begin{array}{l}\text { Forêts “pilotes" } \\
\text { (modalité mixte 1) }\end{array}$} \\
\hline & $\mathrm{T}_{1}$ & $\mathrm{~T} 2$ & $\mathrm{~T}_{3}$ & Risquetout & Organabo \\
\hline 4 ans après traitement sylvicole $\ldots$. & 1,9 & 2,8 & 2,7 & 1,6 & 1,3 \\
\hline 12 ans après traitement sylvicole . . . & 1,8 & 2,9 & 3,0 & - & - \\
\hline
\end{tabular}

Il n'est cependant pas possible de comparer directement les résultats des 3 dispositifs, qui connaissent des conditions environnementales différentes (pluviométrie et géologie en particulier), et dont les peuplements n'avaient pas la même composition floristique de départ. De plus, la dynamique des parcelles de Risquetout et d'Organabo était, avant traitement, différente de celle des parcelles de Paracou, du fait de perturbations récentes. Les accroissements diamétriques enregistrés dans les parcelles témoins de ces sites sont tout à fait comparables à ceux que l'on observe à Paracou dans les parcelles exploitées, 10 ans après intervention (0,30 à $0,35 \mathrm{~cm} / \mathrm{an})$. Il apparaît ainsi assez normal que les peuplements des forêts pilotes aient moins réagi à une ouverture supplémentaire que ceux de Paracou qui partaient d'un état de quasi-équilibre.

L'effet de l'ouverture de peuplements initialement non perturbés est donc fort sur l'accroissement en diamètre. Il est durable, puisqu'il est encore mesurable 12 ans après l'intervention sylvicole, même dans le cas d'une exploitation seule (traitement T1). Cet effet augmente avec l'intensité du traitement $\left(T_{1}<T_{2}\right)$ jusqu'à une certaine limite vraisemblablement due à de trop importantes modifications du microclimat dans les parcelles : les accroissements en $T_{3}$ ne sont pas significativement différents des accroissements en T2 (Gourlet-Fleury, 2000).

Enfin, il est possible de relancer significativement la croissance dans des peuplements déjà exploités une dizaine d'années auparavant, comme le montrent les résultats obtenus dans les forêts "pilotes" (Fuhr et Plancheron, 2001). 
L'effet des traitements est variable selon les classes de diamètre considérées : ce sont les arbres les plus petits qui réagissent le plus. Cependant, à Paracou, les arbres de gros diamètre montrent encore une réaction positive aux interventions (cf. figure 4 , ci-dessous).

FIGURE 4

$$
\begin{aligned}
& \text { ACCROISSEMENTS EN DIAMÈTRE PAR CLASSES DE DIAMÈTRE, } \\
& \text { GROUPES DE PARCELLES ET TOUTES ESSENCES CONFONDUES Â PARACOU, } \\
& \text { ENTRE } 4 \text { ANS ET } 7 \text { ANS APRĖS INTERVENTION SYLVICOLE }
\end{aligned}
$$

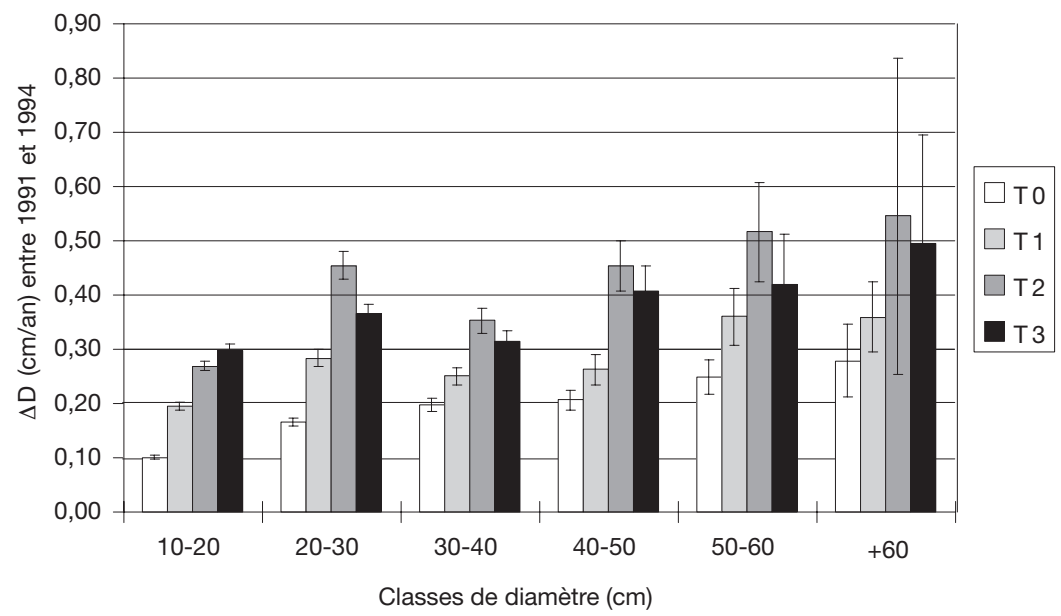

Les moyennes sont représentées avec leur intervalle de confiance à $95 \%$.

Intéressons-nous à nouveau à l'Angélique, aux Gonfolos et au Grignon franc. Les résultats obtenus sur Paracou sont les suivants :

TABLEAU IV Facteur multiplicatif traitement sylvicole sur la croissance en diamètre en fonction de l'intensité du traitement et des essences (données de Paracou, période 1991-1993), nota: To $=1$

\begin{tabular}{|l|c|c|c|}
\hline & $T_{1}$ & $T_{2}$ & $T_{3}$ \\
\hline Angélique $\ldots \ldots \ldots \ldots \ldots \ldots$ & 1,6 & 1,6 & 1,4 \\
\hline Gonfolos $\ldots \ldots \ldots \ldots \ldots \ldots \ldots \ldots \ldots \ldots \ldots \ldots$ & 1,6 & 2,5 & 2,3 \\
\hline Grignon $\ldots \ldots \ldots \ldots$ & 1,9 & 3,0 \\
\hline
\end{tabular}

L'effet complémentaire de l'éclaircie par rapport à la seule exploitation de gros bois (comparaison entre $\mathrm{T}_{1}$ et $\mathrm{T}_{2}$ ) est variable selon les essences : il est très léger pour l'Angélique, tandis qu'il est sensible pour les Gonfolos et le Grignon franc. L'existence d'un plafond de stimulation n'apparaît pas dans le cas du Grignon franc, puisque l'effet multiplicatif est très marqué entre le traitement $\mathrm{T}_{2}$ et le traitement T3. Toutefois, les effectifs des Grignons étant faibles, il est difficile de conclure sur ce point.

Globalement, l'exploitation forestière de gros bois et les éclaircies ont un effet stimulant sur l'accroissement en diamètre du peuplement. Cet accroissement est variable suivant les classes de diamètre et les essences. 


\section{Effet des interventions sylvicoles sur la mortalité naturelle}

Un autre paramètre couramment suivi dans les dispositifs est la mortalité des arbres, naturelle ou directement liée aux traitements sylvicoles (casse dans le voisinage des arbres exploités, mort par empoisonnement).

Dans les forêts "pilotes" de Risquetout et Organabo, les peuplements témoins (zones exploitées fin 1980-début 1990) montrent un taux de mortalité naturelle annuel voisin de $1 \%$ (taux de mortalité calculés sur 4 ans, voir tableau V, ci-dessous). Ce taux est plus faible pour le peuplement désigné(5), les individus étant a priori sains et vigoureux.

tABleau V Effet de l'éclaircie mixte 1 sur le taux de mortalité naturelle annuel des peuplements commercial et désigné

(données de Risquetout parcelle 5 et Organabo parcelle 6, 4 ans après intervention)

\begin{tabular}{|l|c|c|c|c|}
\hline \multirow{2}{*}{} & \multicolumn{2}{|c|}{ Risquetout } & \multicolumn{2}{c|}{ Organabo } \\
\cline { 2 - 4 } & témoin & éclaircie & témoin & éclaircie \\
\hline Peuplement commercial . . . & $0,99 \%$ & $2,55 \%$ & $1,05 \%$ & $1,83 \%$ \\
\hline Peuplement désigné . . . . . & $0,69 \%$ & $2,5 \%$ & $0,28 \%$ & $1,25 \%$ \\
\hline
\end{tabular}

À titre de comparaison, les données de Paracou sur la période 1988-1989 à 1991-1992, soit pendant le pic de mortalité le plus important suivant les traitements, sont fournies dans le tableau VI (ci-dessous) :

TABLEAU VI Effet de l'éclaircie mixte 1 sur le taux de mortalité naturelle annuel du peuplement commercial

(données de Paracou sur la période 1988-1989 à 1991-1992)

\begin{tabular}{|c|c|c|c|c|}
\hline & To & $\mathrm{T}_{1}$ & $\mathrm{~T} 2$ & T3 \\
\hline $\begin{array}{l}\text { Peuplement commercial } \ldots \ldots \ldots \ldots \ldots \ldots \\
\text { (58 espèces et groupes d'espèces) }\end{array}$ & $0,88 \%$ & $1,08 \%$ & $1,92 \%$ & $1,62 \%$ \\
\hline
\end{tabular}

On observe, après les traitements, une déstabilisation importante du peuplement qui s'est étalée, dans le cas de Paracou, sur 4 à 5 ans. Le taux de mortalité du peuplement commercial est ainsi multiplié par un facteur allant de 1,2 à 2,2. Cette surmortalité (hors mortalité directe liée aux traitements) provient :

- de la mort des arbres blessés dans les années suivant l'intervention,

- d'une augmentation sensible des chablis primaires,

- d'une augmentation des chablis secondaires liée à la chute des arbres dévitalisés.

Pour les arbres d'avenir, le taux de mortalité est multiplié par un facteur 3 à 4 sur les forêts "pilotes". Cette déstabilisation du peuplement va en partie masquer les effets positifs de l'éclaircie sur la croissance et le recrutement (Fuhr et Plancheron, 2001).

Par la suite, dans la plupart des dispositifs, le taux de mortalité revient à des valeurs raisonnables, légèrement supérieures cependant aux valeurs observées dans une forêt non perturbée. Trois à quatre ans après l'intervention, il n'apparaît pas de lien net entre l'intensité de l'éclaircie

(5) Par peuplement désigné, on entend les arbres au profit desquels est réalisée l'intervention sylvicole. 
et le taux de mortalité du peuplement. Dans le cas de Paracou, il faut attendre au moins 8 ans pour voir un retour aux valeurs observées dans les témoins.

\section{Effet des interventions sylvicoles sur le recrutement}

Le recrutement correspond au nombre d'arbres qui franchissent chaque année le seuil de précomptage de $10 \mathrm{~cm}$ de diamètre. Il est donc lié à l'accroissement en diamètre des individus qui constituent la "régénération" au sens large. Les données obtenues sur les forêts "pilotes" montrent une stimulation nette du recrutement des espèces commerciales (cf. tableau VII, ci-dessous) après traitement. L'intensité de l'ouverture du couvert et le recrutement sont corrélés comme tend à le montrer la figure 5 (ci-dessous). Plus l'ouverture est importante, plus le recrutement est fort.

TABLEAU VII Effet de l'éclaircie mixte 1 sur le recrutement du peuplement commercial

\begin{tabular}{|l|c|c|}
\hline & Témoin & \multicolumn{1}{c|}{ Éclaircie } \\
\hline Risquetout $\ldots \ldots \ldots \ldots \ldots$ & 1,7 tige/ha/an & 3,6 tiges/ha/an \\
\hline Organabo $\ldots \ldots \ldots \ldots$ & 4,5 tiges/ha/an & 6,4 tiges/ha/an \\
\hline
\end{tabular}

FIGURE 5

EFFET DE L'INTENSITÉ DE L'INTERVENTION SYLVICOLE SUR LE RECRUTEMENT (données de Paracou)

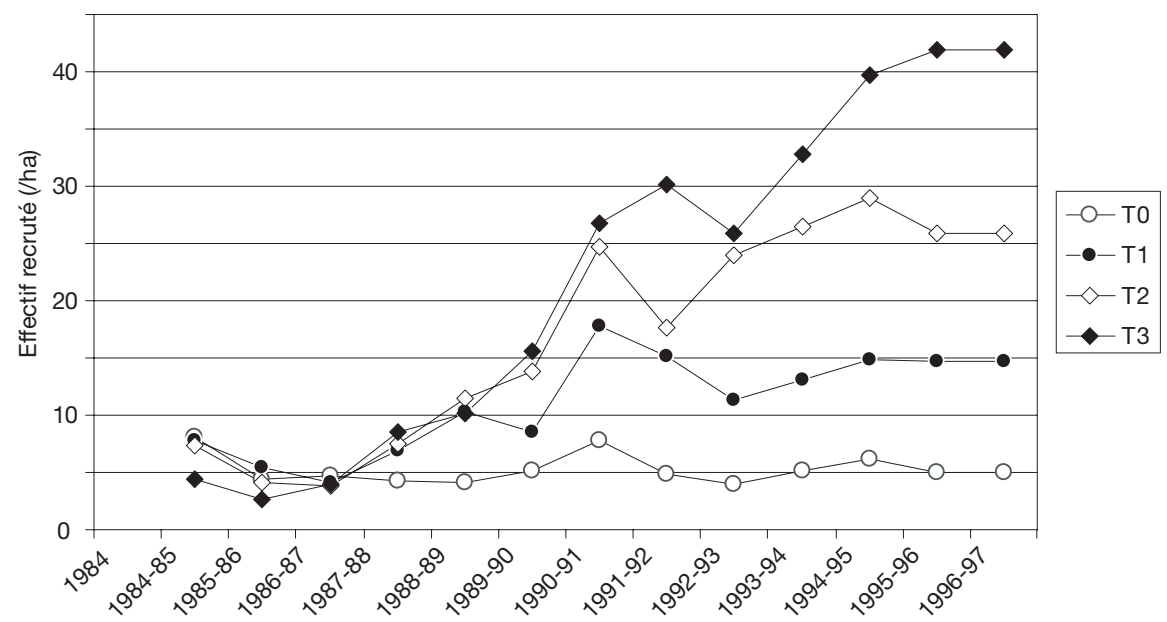

NB : La dépression de 1994-1995 est liée à un artefact d’inventaire.

Cependant, cette ouverture n'est pas sans conséquence sur la composition floristique. Le recrutement des essences héliophiles colonisatrices est favorisé par les ouvertures importantes : à Paracou, quatre espèces pionnières quasiment absentes des parcelles témoins (Cecropia obtusa Trec et Cecropia sciadophylla Martius, Moraceae, Vismia cayennensis (N.J. Jacquin) Persoon, Clusiaceae, et Tapirira guianensis Aublet, Anacardiaceae) représentent, 8 ans après application des traitements, $30 \%$ du recrutement en $\mathrm{T}_{2}$ et presque $50 \%$ en $\mathrm{T}_{3}$.

Si ces espèces ont bien souvent une durée de vie courte, il n'en reste pas moins que la composition floristique des peuplements traités restera affectée à moyen terme par la plus grande place 
prise par les espèces héliophiles à durée de vie longue (Favrichon, 1997), peu intéressantes à l'heure actuelle pour l'exploitation en Guyane.

\section{Bilan et préconisations pour des interventions sylvicoles en Guyane}

En résumé, les essais d'éclaircie en forêt tropicale humide guyanaise ont montré les effets suivants, perceptibles dans la décennie qui suit l'intervention et s'atténuant au-delà :

- un gain d'accroissement sur le diamètre des espèces commerciales pendant une dizaine d'années au moins ; l'accroissement en diamètre est multiplié en moyenne par un facteur de 1,5 à 2 ;

- la réaction des arbres est d'autant plus importante et rapide que les individus sont petits ; l'essentiel du gain de croissance en volume est enregistré dans le peuplement d'avenir $(10 \mathrm{~cm} \leq \mathrm{D}<50 \mathrm{~cm})$;

- une mortalité induite importante du peuplement pendant les 4 à 8 premières années suivant l'intervention, selon son intensité ; ensuite, la mortalité rejoint des valeurs proches de celles d'une forêt non perturbée ;

- une stimulation du recrutement des espèces héliophiles.

L'enseignement tiré du suivi des dispositifs sylvicoles expérimentaux permet d'établir les recommandations suivantes en matière d'interventions :

- ne pas dépasser un certain seuil d'ouverture du couvert afin de limiter la prolifération des espèces pionnières sans intérêt commercial et des lianes. En pratique, les résultats obtenus à Paracou montrent qu'il n'est pas souhaitable d'ouvrir le peuplement davantage qu'il ne l'a été en T2, c'est-à-dire d'enlever plus d'un tiers de la surface terrière du peuplement initial.

- éviter le recours aux éclaircies systématiques, sauf dans les peuplements riches en tiges d'avenir, et préférer dans la mesure du possible les éclaircies sélectives, pour une meilleure prise en compte de la biodiversité.

Si l'objectif de ces études est, à ce stade, de mieux comprendre le fonctionnement écologique de la forêt tropicale humide de Guyane après une exploitation forestière, il semble toutefois intéressant de fournir quelques éléments économiques.

Les essais menés sur les forêts "pilotes" de Risquetout et d'Organabo font ressortir des coûts d'intervention de 150 à 300 euros/ha pour la modalité mixte 2 et de 500 à 800 euros/ha pour la modalité mixte 3 .

Même si ces données sont ponctuelles, il est intéressant de les mettre en relation avec le coût du bois sur pied ${ }^{(6)}$ (46 euros $/ \mathrm{m}^{3}$ grume), la faiblesse du prix de vente moyen du bois sur pied (9,3 euros $/ \mathrm{m}^{3}$ grume) et du prélèvement moyen à l'hectare $\left(5,5 \mathrm{~m}^{3} / \mathrm{ha}\right)$. Il apparaît ainsi que, dans les conditions actuelles, la mise en œuvre de l'éclaircie mixte 2 coûterait de 27 à 54 euros $/ \mathrm{m}^{3}$, soit de 60 à $110 \%$ du coût actuel du bois sur pied !

À l'avenir, il semble toutefois indispensable d'associer une approche économique à cette approche écologique des interventions sylvicoles.

(6) Le coût du bois sur pied est la somme des coûts suivants : aménagements forestiers, programmation des travaux, diagnostics et inventaires, réalisation de pistes forestières, organisation des ventes de bois et contrôle des exploitations. Ce coût est assumé pour partie par l'ONF (50\%) et pour partie par l'Europe et l'État. 


\section{Simulations de la reconstitution des peuplements : étude de cas à Paracou}

À partir des données de Paracou, un certain nombre de simulations de "reconstitution" (7) du stock exploitable ont été réalisés. Le travail a porté sur un ensemble de 17 espèces ou groupes d'espèces actuellement les plus recherchées en Guyane, au nombre desquelles l'Angélique, les Gonfolos, le Grignon franc et le Wacapou.

Quatre scénarios différents, combinant mode d'intervention et hypothèses haute et basse sur la mortalité naturelle (cf. tableau VIII, ci-dessous) ont été simulés. On a en particulier envisagé le cas d'interventions régulières en éclaircie (passage tous les 10 ou 15 ans par exemple) qui auraient pour effet de maintenir les accroissements au niveau observé en T1.

TABLEAU VIII

\section{Explicitation des scénarios utilisés pour simuler la reconstitution du stock exploité}

(Gourlet-Fleury, 2000)

\begin{tabular}{|c|c|c|}
\hline & Mortalité observée en T1 & $\begin{array}{c}\text { Mortalité maximale observée } \\
\text { sur le site }\end{array}$ \\
\hline Exploitation seule $\ldots \ldots \ldots \ldots$ & $\begin{array}{l}\text { Scénario } 1 \\
\text { Stimulation des accroissements } \\
\text { pendant } 10 \text { ans, puis retour } \\
\text { aux accroissements du témoin }\end{array}$ & $\begin{array}{l}\text { Scénario } 2 \\
\text { Stimulation des accroissements } \\
\text { pendant } 10 \text { ans, puis retour } \\
\text { aux accroissements du témoin }\end{array}$ \\
\hline $\begin{array}{l}\text { Exploitation }+ \text { éclaircies } \\
\text { régulières } \ldots \ldots \ldots \ldots \ldots\end{array}$ & $\begin{array}{l}\text { Scénario } 3 \\
\text { Stimulation constante des } \\
\text { accroissements }\end{array}$ & $\begin{array}{l}\text { Scénario } 4 \\
\text { Stimulation constante des } \\
\text { accroissements }\end{array}$ \\
\hline
\end{tabular}

Le stock exploitable, au-delà du diamètre minimum d'exploitation (DME) pour les 17 espèces ou groupes d'espèces, varie entre 4,2 et 5,5 tiges/ha en début de simulation.

Les résultats des simulations, d'un point de vue quantitatif, sont les suivants (Gourlet-Fleury, 2000):

- scénario 1 ("le plus raisonnable") : le stock n'est reconstitué en moins de 50 ans que dans les parcelles les moins riches au départ ; il faudrait attendre entre 90 et 100 ans sur les parcelles les plus riches, dans lesquelles la diminution du stock a été plus importante, sachant toutefois que la fiabilité des prédictions à cette échelle de temps est limitée ;

- scénario 2 (“le plus pessimiste") : il faudrait 50 à 60 ans pour reconstituer le stock des parcelles les moins riches, soit 10 ans de plus que dans le premier scénario ; pour les parcelles les plus riches, seule la moitié du stock serait récupérée après 50 ans (2,5 à 2,9 tiges/ha) ;

- scénario 3 : ce scénario simule le cas de parcelles qui demeurent relativement ouvertes, grâce à des éclaircies régulières ; l'effet sur l'évolution du stock est très net ; un gain de 10 à 15 ans serait possible sur les parcelles les moins riches, tandis que les parcelles les plus riches pourraient voir leur stock entièrement reconstitué en moins de 60 ans ;

- scénario 4 : en fixant les taux de mortalité à leur maximum, les résultats évoluent peu. Trente ans permettent de reconstituer le stock des parcelles les moins riches; il faudrait 60 ans dans le cas des parcelles les plus riches pour retrouver entre $70 \%$ et $90 \%$ du stock initial.

Il faut souligner l'importance du rôle joué par la mortalité naturelle. Dans un grand nombre des simulations effectuées espèce par espèce, le stock des arbres exploitables passe par un maximum inférieur à l'effectif initial, avant de décroître. Ceci est dû au fait que le recrutement au-dessus du DME est insuffisant pour compenser les pertes dues à la mortalité naturelle.

(7) Par "reconstitution" du stock, on entend un retour à $100 \%$ de l'effectif de départ, avant exploitation. 
D'un point de vue qualitatif, il est utile de garder à l'esprit que la composition floristique spécifique du peuplement exploité en deuxième rotation sera différente de celle du peuplement initialement exploité. À Paracou, certaines espèces ne retrouveront pas leurs effectifs initiaux (Grignon, Chawari, Cœur dehors, Ebène verte par exemple), tandis que d'autres au contraire peuvent voir leurs effectifs augmenter (Carapa, Wacapou, Goupi, Balata franc). L'évolution des effectifs par essence dépend des structures diamétriques spécifiques initiales, qui peuvent être très variables d'un secteur à l'autre, même sur une superficie réduite comme celle de Paracou. Dans ces conditions, seuls le recul dans le temps et l'accumulation d'observations sur l'autécologie des espèces permettront d'ajuster ces résultats et de moduler les diamètres minimum d'exploitation et les durées de révolution en fonction des essences objectifs et des données d'inventaire avant exploitation.

Sur un site identique à celui de Paracou, les préconisations suivantes en termes d'exploitation des peuplements peuvent finalement être faites :

- exploiter l'intégralité du stock initial des arbres bien conformés, y compris lors du premier passage en exploitation, ceci afin d'éviter de perdre des individus par mortalité naturelle ; pour certaines espèces comme le Grignon ou le Chawari dont le renouvellement des effectifs exploitables risque d'être très long, il paraît préférable là encore d'exploiter le stock existant quitte à suspendre l'exploitation de ces essences par la suite ; cette préconisation peut être modulée par des règles de précaution relevant de la préservation de la diversité végétale et animale (préservation de semenciers, d'abris, de nourriture...) ;

- éviter les durées de rotation trop longues, pour deux raisons : on risque de perdre des arbres exploitables par mortalité naturelle et l'ouverture des peuplements est le moteur de la croissance ; si aucune éclaircie complémentaire n'est réalisée au cours de la rotation, l'exploitation reste le seul moyen de stimuler la dynamique des peuplements; à Paracou, la durée de rotation la plus adaptée nous paraît être de 50 ans, elle permettrait, en moyenne, la récupération de $87 \%$ du stock initial exploité ;

- prévoir que, en l'absence de sylviculture complémentaire, l'exploitation à la rotation suivante portera sur un effectif plus réduit et, dans tous les cas, sur une répartition différente entre espèces.

\section{EXPLOITATION À FAIBLE IMPACT (EFI)}

Nous avons vu que les éclaircies sélectives, sous certaines conditions, peuvent stimuler la reconstitution du stock d'arbres exploitables. Cependant, cette intervention s'avère coûteuse et peut être difficile à mettre en œuvre. Il convient donc de ne pas oublier que l'exploitation forestière de gros arbres constitue en premier lieu une intervention sylvicole. En l'absence d'éclaircie, l'exploitation constitue l'unique intervention, qui doit alors être répétée plus "fréquemment" (tous les 50 ans plutôt que tous les 70 ans pour citer les chiffres de Paracou), l'idée étant que les peuplements produisent plus de bois lorsqu'ils sont ouverts (Gourlet-Fleury, 2000). Cependant, le corollaire est de réduire autant que possible les dégâts d'exploitation.

Le contrôle de l'exploitation forestière est décisif pour la régénération naturelle et la reconstitution du peuplement adulte. Il apparaît donc fondamental, dans le cadre d'une gestion durable, de mettre en œuvre une exploitation dite à faible impact.

\section{Les acquis des études sur l'EFI}

L'exploitation à faible impact est caractérisée par la mise en œuvre, dans de nombreux pays, de certaines règles élémentaires, dont les principales sont récapitulées dans le tableau IX (p. 286). Ces règles visent à assurer la durabilité de l'exploitation et à conserver la diversité biologique de la forêt. 


\begin{tabular}{|c|c|}
\hline Durabilité de l'exploitation & Conservation de la diversité biologique \\
\hline $\begin{array}{l}\text { - Interdire la repasse (passages répétés en coupe) } \\
\text { en cours de rotation } \\
\text { - Limiter le délai d'exploitation d'une parcelle ( } 3 \text { ans } \\
\text { maximum) } \\
\text { - Fixer un seuil maximum de prélèvement afin de } \\
\text { limiter les dégâts d'exploitation } \\
\text { - Optimiser le réseau de débardage par une } \\
\text { cartographie des arbres à abattre } \\
\text { - Orienter, ou du moins contrôler l'abattage } \\
\text { - Utiliser l'égobelage }{ }^{(1)} \text { afin de valoriser au mieux } \\
\text { les arbres abattus }\end{array}$ & $\begin{array}{l}\text { - Concentrer l'exploitation dans l'espace et dans le } \\
\text { temps, en particulier pour limiter les impacts sur la } \\
\text { faune sauvage } \\
\text { - Préserver les écosystèmes fragiles ou à intérêt } \\
\text { écologique particulier en y interdisant l'exploitation } \\
\text { forestière } \\
\text { - Exploiter avec prudence les espèces dont la } \\
\text { structure diamétrique est fortement déséquilibrée vers } \\
\text { les gros bois (préservation de semenciers, d'abris, de } \\
\text { nourriture) } \\
\text { - Conserver les espèces rares, endémiques, } \\
\text { ressources-clés }\end{array}$ \\
\hline
\end{tabular}

(1) L'égobelage consiste à éliminer les contreforts avant l’abattage.

Bertault et Sist (1997), Van der Hout (1999), Sist (2000) et Chabbert et Priyadi (2001) ont, dans leurs travaux, comparé l'exploitation à faible impact à une exploitation dite conventionnelle en forêt tropicale humide. Retenons les conclusions suivantes :

- les dégâts de débardage sont significativement inférieurs ; la mortalité directement liée au traitement diminue fortement ;

- la surface de canopée perturbée est inférieure à celle d'une exploitation conventionnelle ;

- la surface au sol affectée par les pistes de débuscage et de débardage diminue significativement, voire presque de moitié.

\section{L'exploitation à faible impact en Guyane}

Rappelons tout d'abord que la première caractéristique de l'exploitation forestière en Guyane est la faible intensité des prélèvements : moins de 2 tiges exploitées par hectare, sur un potentiel de 6,5 tiges par hectare en moyenne. Cependant, cette moyenne cache une forte hétérogénéité spatiale et, localement, l'exploitation peut atteindre des intensités proches de celles observées à Paracou. Ceci justifie pleinement la mise en œuvre de règles d'exploitation à faible impact.

Ainsi, le cahier des charges des exploitants forestiers inclut les règles suivantes :

- le délai d'exploitation des bois d'une parcelle est généralement fixé à deux saisons sèches pour valoriser au mieux les essences présentes sur la coupe ;

- des repères indiquant la direction et le nombre des arbres abattus par les bûcherons doivent être systématiquement mis en place à l'intention du débardeur ;

- la coupe ne doit pas se faire à plus d'un mètre de hauteur, mesurée à partir du point le plus haut du sol ; de plus, les grumes doivent être égobelées ;

- l'abattage directionnel doit être privilégié ;

- les arbres réservés doivent être préservés de la coupe et des autres dégâts d'exploitation ;

- le cubage des grumes doit permettre de dénombrer les arbres exploités ; le numérotage de billons devra donc faire référence au numéro de la grume ; ceci permettra de mieux connaître les prélèvements en forêt, non seulement en volume, mais en nombre d'arbres, et de pouvoir les comparer avec les résultats de l'inventaire avant exploitation ;

- l'acheteur doit mettre en place un dispositif de contrôle du débardage effectif de l'ensemble des grumes exploitées (numérotation simultanée des arbres à la souche et sur la grume, ou tout autre système à proposer par l'exploitant et à faire agréer par l'ONF) ;

- le nombre et la localisation des parcs à grumes sont fixés pour limiter les déforestages. 
Le cahier des charges ${ }^{(8)}$ comprend en outre d'autres règles applicables à toute exploitation conventionnelle en zone tropicale, telles que les suivantes:

- sauf impossibilité, les arbres abattus ne devront pas tomber sur les routes forestières, afin notamment d'éviter les saignées créées par la chute et l'engorgement des fossés par les houppiers ;

- les vidanges sauvages des engins en forêt sont interdites, ainsi que l'abandon des déchets non végétaux après la fin de l'exploitation ;

- la remise en état des routes forestières après l'exploitation est à la charge de l'acheteur, et notamment pour ce qui concerne les fossés bouchés, les ravinements occasionnés par les chenilles de bulldozer ;

- les barrières de pluie doivent être respectées afin de préserver l'état des routes forestières.

Il faut noter que, à l'heure actuelle, les études relatives à l'impact de l'exploitation forestière en Guyane sont peu nombreuses et très localisées. L'ONF et le CIRAD-Forêt ont engagé en 2002 un projet sur l'évaluation des dégâts d'exploitation afin d'obtenir une meilleure connaissance des conditions de réalisation de l'exploitation par les entreprises.

Les premiers résultats, forcément partiels, mettent toutefois en évidence des points positifs comme :

- les arbres marqués en réserve sont respectés ;

- les zones interdites à l'exploitation (par exemple, les anciennes zones d'habitation amérindiennes) sont respectées ;

- la densité de pistes de débuscage et de débardage est relativement faible, donc peu perturbante [9 km de piste/100 ha, ce qui est comparable aux chiffres mentionnés par Chabbert et Priyadi (2001)], mais qui représente des surfaces au sol affectées importantes au regard des volumes exploités (environ $35 \mathrm{~m}^{2} / \mathrm{m}^{3}$ bois extrait) (cf. photo 2, p. 288) ;

... et des points à améliorer pour se rapprocher d'une exploitation réellement à faible impact, comme :

- l'absence effective de localisation des arbres abattus, ce qui engendre des abandons de grumes en forêt (de l'ordre de $5 \%$ des arbres exploités) (Cuypers et al., 2002) ;

- un égobelage jamais pratiqué et des purges excessives qui représenteraient $12 \%$ du volume exploité ;

- l'absence de mise en place d'un système de contrôle du débardage ;

- des techniques d'abattage directionnel peu souvent pratiquées, malgré la maîtrise des techniques.

Aussi, ce projet devrait permettre de mettre en place un meilleur contrôle du respect des règles d'exploitation à faible impact, mais aussi de modifier le cahier des charges des exploitants et de garantir une plus grande durabilité de l'exploitation. Un vaste champ d'investigation demeure notamment dans le contrôle de la taille des trouées d'abattage et la maîtrise de la régénération (semenciers, agrégats, plaques, semis...).

Toutefois, comme le soulignent, à juste titre, Chabbert et Priyadi (2001), seules les analyses économiques sont aujourd'hui en mesure de convaincre les exploitants des bénéfices qu'ils peuvent tirer de la mise en œuvre de techniques à faible impact. Homes et al. (2002) montrent que les coûts d'exploitation sont réduits de $12 \%$ dans le cas de l'exploitation à faible impact, en comparaison à une exploitation conventionnelle. Si les coûts de planification sont plus élevés, la diminution des pertes de bois en forêt permet de réduire les coûts au volume sorti.

(8) Le cahier des charges est un document contractuel entre l'ONF et les entreprises d'exploitation forestière. Ce document est établi par la Direction régionale de l'ONF en Guyane et révisé annuellement. 


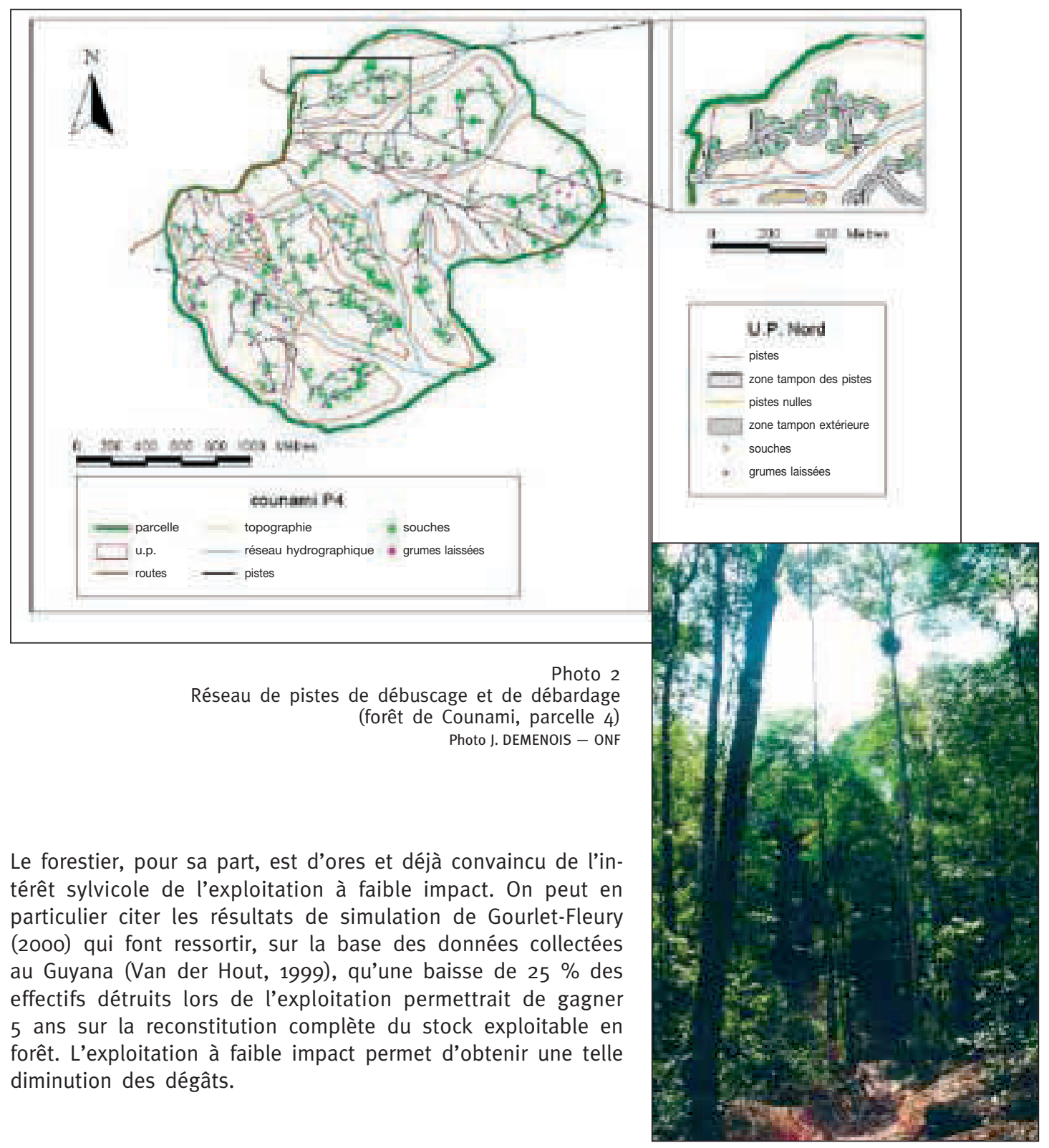

\section{CONCLUSIONS}

La sylviculture en forêt tropicale humide concerne l'exploitation forestière et les éclaircies postexploitation. Pour évaluer correctement son impact sur la dynamique des peuplements et la reconstitution des stocks exploitables, il est indispensable de suivre sur le long terme les dispositifs de recherche comme les placettes permanentes.

L'effet positif des éclaircies sélectives, sous certaines conditions, et les effets stimulants de l'exploitation forestière des gros bois sur la croissance des peuplements ont été montrés. Toutefois, 
il demeure indispensable de poursuivre l'installation de nouvelles placettes, afin de couvrir des situations environnementales diverses et d'extrapoler avec plus de fiabilité les résultats obtenus à Paracou, Risquetout ou Organabo à l'ensemble du massif forestier aménagé de Guyane.

De plus, une caractérisation plus fine de l'exploitation, notamment en termes de dégâts d'exploitation et de régénération au niveau des places d'abattage, s'avère nécessaire pour la définition de nouvelles règles d'exploitation à faible impact et leur mise en application effective en Guyane.

Julien DEMENOIS

OFFICE NATIONAL DES FORÊTS

Réserve de Montabo

BP 7002

F-97307 CAYENNE CEDEX

(julien.demenois@onf.fr)

Marc FUHR

OFFICE NATIONAL DES FORÊTS

Rue Georges-Ponsot

BP 54

F-71001 VESOUL CEDEX

(marc.fuhr@onf.fr)
Sylvie GOURLET-FLEURY

CIRAD-Forêt

TA 10/D

Campus international de Baillarguet

F-34398 MONTPELLIER CEDEX 5

(sylvie.gourlet-fleury@cirad.fr)

BIBLIOGRAPHIE

BERTAULT (J.-G.), SIST (P.). - An experimental comparison of different harvesting intensities with reducedimpact and conventional logging in East Kalimantan, Indonesia. - Forest Ecology and Management, vol. 94, 1997, pp. 209-218.

CATINOT (R.). - Sylviculture tropicale en forêt dense africaine. - Bois et Forêts des Tropiques, $\mathrm{n}^{\circ} 100$, 1965a, pp. 5-18.

CATINOT (R.). - Sylviculture tropicale en forêt dense africaine (suite). - Bois et Forêts des Tropiques, $\mathrm{n}^{\circ} 101,1965 \mathrm{~b}, \mathrm{pp} .3-16$.

CHABBERT (J.), PRIYADI (H.). - Exploitation à faible impact dans une forêt de Bornéo. - Bois et Forêts des Tropiques, $\mathrm{n}^{\circ}$ 269, 2001, pp. 82-86.

CUYPERS (D.), GLATTARD (E.), LEJONC (G.), ORIOL (E.). - Élaboration d'une méthode de diagnostic postexploitation à partir du cas de la forêt de Counami (Guyane française). - Rapport ENGREF-module FTH, 2002. - 17 p. + annexes.

DUPUY (B.). - Bases pour une sylviculture en forêt dense tropicale humide africaine. - CIRAD-Forêt ; Coopération française ; CIFOR, 1998. - 328 p. (Série FORAFRI, document $n^{\circ} 4$ ).

DURRIEU DE MADRON (L.), FORNI (E.), MEKOK (M.). - Les techniques d'exploitation à faible impact en forêt dense humide camerounaise. - CIRAD-Forêt ; Coopération française ; CIFOR, 1998. - 28 p. (Série FORAFRI, document $\left.\mathrm{n}^{\circ} 17\right)$.

FAVRICHON (V.). - Réaction de peuplements forestiers tropicaux à des interventions sylvicoles. - Bois et Forêts des Tropiques, $\mathrm{n}^{\circ}$ 254, 1997, pp. 5-24.

FUHR (M.), PLANCHERON (F.). - Forêts "pilotes" de Risquetout et Organabo - Éclaircie mixte modalité 1 Aperçu sur la dynamique des peuplements 4 ans après intervention. - ONF, 2001. -25 p. + annexes. 
GOURLET-FLEURY (S.). - Dispositif sylvicole expérimental de Paracou (Guyane française). Aperçus sur l'état et la dynamique des peuplements 13 ans après installation. Rapport interne CIRAD/Silvolab-Guyane, 2000. -253 p. + annexes.

GOURLET-FLEURY (S.). - Simulation par l'ONF et le CTFT d'une éclaircie sélective sur une parcelle exploitée (parcelle 7) du dispositif de Paracou. - Rapport interne CTFT, 1990. - $11 \mathrm{p}$.

GOURLET-FLEURY (S.). - Simulation d'éclaircies sur le dispositif sylvicole de Paracou (Guyane française) : recherche de méthodes d'intervention en forêt, après exploitation. - IASBSE - Université Claude Bernard - Lyon I, 1992. - 34 p. + annexes (Mémoire technique - DEA d'Analyse et de Modélisation des Systèmes biologiques).

HOMES (T.P.), BLATE (G.M.), ZWEEDE (J.C.), PEREIRA JUNIOR (R.), BARRETO (P.), BOLTZ (F.). - Custos e beneficios financeiros da exploraçao florestal de impacto reduzido em comparaçao à exploraçao florestal convencional na Amazönia Oriental. - Belém : Fundaçao Floresta Tropical, 2002. - 69 p.

PINARD (M.A.), PUTZ (F.E.), TAY (J.), SULLIVAN (T.E.). - Creating timber harvest guidelines for a reducedimpact logging project in Malaysia. - Journal of Forestry, vol. 93, n ${ }^{\circ}$ 10, 1995, pp. 41-45.

SIST (P.). - Les techniques d'exploitation à faible impact. - Bois et Forêts des Tropiques, vol. 265, 2000, pp. 31-43.

VAN DER HOUT (P.). - Reduced impact logging in the tropical rain forest of Guyana. Ecological, economic and silvicultural consequences. - Tropenbos-Guyana Series 6, 1999, 335 p.

VAN LEERSUM (G.J.R.), NGIBAOT (F.), LAAN (E.A.), JONKERS (W.B.J.). - Logging methods applied in South Cameroon and ways for their improvement. In : Sustainable Management of African Rain Forest, Part II : Symposium / W.B.J. Jonkers, B. Foahom, P. Schmidt Eds. - Proceedings of a seminar held in Kribi, Cameroon, November 1999. - 2001. - pp. 82-88.

\section{SYLUICULTURE EN FORÊT TROPICALE HUMIDE GUYANAISE [Résumé]}

Cet article vise à montrer que, depuis les premiers essais de sylviculture interventionniste en forêt tropicale humide, les approches ont évolué et ont permis de mettre en lumière les réactions des peuplements forestiers à certains types d'actions sylvicoles : exploitation forestière et éclaircie.

L'effet stimulant de ces interventions sur la dynamique forestière et sur la reconstitution des stocks exploitables en forêt a été montré sur tous les dispositifs expérimentaux installés en forêt tropicale, et en particulier sur ceux de Guyane. II existe cependant des limites : l'ouverture excessive, que l'on a évalué à Paracou à l'équivalent de l'élimination de plus de un tiers de la surface terrière initiale, n'a plus d'effet sur la croissance tandis qu'elle favorise l'invasion des peuplements par des espèces pionnières et héliophiles sans intérêt commercial ; l'effet des ouvertures est limité dans le temps d'où l'intérêt, lorsque l'on ne peut pratiquer d'éclaircie complémentaire aux exploitations, de ne pas opter pour des durées de rotation trop longues. Il est alors crucial d'utiliser l'exploitation comme un véritable outil sylvicole et en particulier de limiter au maximum les dégâts causés au peuplement résiduel : les auteurs présentent les enjeux et les modalités de l'exploitation à faible impact pour la Guyane.

\section{SILUICULTURAL PAACTISES IN THE TROPICAL RAIN FORESTS OF FRENCH GUIANR [Abstract]}

The aim of this article is to show how approaches have evolved since the first silvicultural experimentations in tropical rainforests. The dynamics of the forest is now better understood, as well as its reaction to logging and thinning.

The stimulating effect of those operations on forest dynamics and the recovery of the timber stock have been demonstrated in all the experimental plots settled in tropical forests, and in particular those of French Guiana. However, limitations have become apparent : (i) excessive canopy opening, which in the case of the Paracou experiments amounted to eliminating more than one third of the initial basal area, has no additional effect on diameter increments while it encourages stand invasion by pioneer and heliophilous species that have no market value ; (ii) the stimulating effect of openings is limited in time when post-logging thinning or liberation is not economically feasible, as is the case in French Guiana, felling cycles should not be too long. Hence it is of primary importance to use logging operations as a true silvicultural tool and contain the damage to the future crop trees left in the stands - the authors describe the implications and possible rules for implementing Reduced Impact Logging for the forests of French Guiana. 